\title{
Efecto de la Tostión del Café (Coffea arabica L. var. Castillo) sobre el Perfil de Taza, Contenido de Compuestos Antioxidantes y la Actividad Antioxidante
}

\author{
Félix O. Díaz ${ }^{1 *}$, Angela M. Ormaza ${ }^{1}$ y Benjamín A. Rojano ${ }^{2}$ \\ (1) Universidad de Caldas. Apartado Postal 275, Colombia (e-mail: felix.diaz@ucaldas.edu.co; \\ angela.ormaza@ucaldas.edu.co \\ (2) Facultad de Ciencias, Universidad Nacional de Colombia, sede Medellín. Apartado postal 2050034, \\ Colombia (e-mail: brojano@unal.edu.co
}

${ }^{*}$ Autor a quien debe ser dirigida la correspondencia

Recibido Oct. 31, 2017; Aceptado Ene. 2, 2018; Versión final Feb. 27, 2018, Publicado Ago. 2018

\begin{abstract}
Resumen
En este estudio, se determinó la retención de metabolitos antioxidantes, su actividad antioxidante y el perfil de taza, de muestras tratadas a diferentes tiempos a una misma temperatura y potencia del tostador. La tostión es un proceso de transformación fisicoquímico aplicado a los granos de café verde con el fin de realzar sus cualidades sensoriales y nutracéuticas. El perfil sensorial del café tostado entre 5,5 y 6,5 min alcanzó la calificación de un patrón excelso al evaluar 7 atributos sensoriales, además, de poseer una alta retención de compuestos antioxidantes (fenoles totales, flavonoides y taninos condensados) y una importante capacidad antioxidante. En consecuencia, se demostró que los cafés denominados como de tostión media poseen atributos sensoriales óptimos combinados con propiedades nutracéuticas destacadas.
\end{abstract}

Palabras clave: café, catación; antioxidantes; torrefacción; taza; alimento nutracéutico

\section{Effect of Coffee Roasting (Coffea arabica L. var. Castillo) on Cup Profile, Antioxidant Compound Content and Antioxidant Activity}

\begin{abstract}
In this study, the retention of antioxidant metabolites, their antioxidant activity and the cup profile of roasted samples at different times at the same temperature and power of the toaster were determined. Roasting is a process of physicochemical transformation applied to coffee beans in order to enhance their sensory and nutraceutical qualities. The sensory profile of roasted samples between 5.5 and 6.5 min reached the qualification of an excelso pattern when evaluating 7 sensorial attributes, in addition, of having a high retention of antioxidant compounds (total phenols, flavonoids and condensed tannins) and an important antioxidant capacity. Consequently, it was shown that coffees referred to as medium roast have optimal sensory attributes combined with highlighted nutraceutical properties.
\end{abstract}

Keywords: coffee, cupping; antioxidants; roasting; cup; nutraceutical food 


\section{INTRODUCCIÓN}

La agrocadena del café es una de las más importantes de la economía nacional (FNCC, 2014) y es generadora de numerosas empresas tostadoras en gran parte del territorio colombiano, dado que cada tostador puede producir perfiles definidos de taza de acuerdo al control de los parámetros de tostión (Arango, 2012). Esto implica que el proceso de tostión, constituye un paso crítico para obtener una buena calidad de taza y es considerado dentro del gremio cafetero como un arte. Son numerosos los estudios que han reportado la capacidad antioxidante del café y sus subproductos, como agentes funcionales que ayudan en la prevención de numerosas enfermedades de carácter crónico y degenerativo (Aguiar et al., 2016; CanoMarquina et al., 2013; Farah, 2012). El café, las frutas, los granos y las verduras son considerados alimentos con capacidad antioxidante, y su consumo debe ser frecuente con el objetivo de minimizar el estrés oxidativo, iniciador de muchas enfermedades como el Parkinson y diferentes tipos de cáncer (Aguiar et al., 2016; Oroian y Escriche, 2015; Yashin et al., 2013). Actualmente, la oferta de la industria cafetera ha generado varias alternativas de consumo de acuerdo a la variedad del café, la curva de tostión desarrollada y el método de preparación aplicado (Illy, 2017).

Los granos de café verde tienen sabor y aroma herbal, siendo necesario su procesamiento térmico por tostión para hacerlo apto para el consumo y liberar su aroma y sabor por medio de sustancias químicas como la cafeína, la trigonelina, los ácidos clorogénicos, el ácido cítrico, el ácido acético y el ácido fórmico (De Luca et al., 2016; Nguyen y Byun, 2013). El proceso de tostión comprende 4 etapas: Secado, desarrollo de aroma y color, descomposición química y estructural y tostado completo. Para obtener tostados uniformes se debe partir de café verde de buena calidad, con humedad uniforme cercana al $12 \%$, tamaño uniforme, color verdoso y brillante característico de un café fresco (FNCC, 2014).Dentro del proceso de tostión, hay liberación de gases de combustión como el dióxido y el monóxido de carbono. Además, se presenta la descomposición de macromoléculas como los carbohidratos, grasas y proteínas. Alrededor de los $140^{\circ} \mathrm{C}$ se presenta la fase de crepitación y descomposición del grano de café, acompañado de un rompimiento estructural debido a las presiones internas generadas por los gases de combustión y vapor de agua que escapan de la estructura. Se presenta un incremento de la porosidad (del 9,8 al 34,2\%) y del volumen (50-80\%) del grano del café (De Luca et al., 2016; Oliveros et al., 2017). A los $200^{\circ} \mathrm{C}$ se generan los compuestos volátiles y no volátiles que generan el olor y sabor del café tostado como productos finales de la reacción de Maillard (Moreira et al., 2017). Por último, se presenta la coloración oscura por producción de melanoidinas como consecuencia de la pirólisis de los azúcares presentes en el café (Coelho et al., 2014).

Las características fisicoquímicas generadas durante la torrefacción o tostión del grano, son causadas por la relación tiempo-temperatura del proceso, donde el sabor desarrollado, el contenido de ácidos clorogénicos y el porcentaje de cafeína, dependen de factores como la variedad cultivada, el beneficio y tipo de tostión aplicado (Gamboa et al., 2013). Dichos componentes, tienen un papel importante en el perfil de taza desarrollado. La relación entre el mecanismo y grado de tostión con las características en taza, no ha sido establecida ni tomada como una oportunidad para ofrecer al mercado variedades de café con características definidas a nivel sensorial y funcional. El objetivo del presente estudio, consiste en determinar la retención de compuestos químicos con capacidad antioxidante junto con el desarrollo de un adecuado perfil de taza durante el proceso de tostión en función del tiempo. Los metabolitos antioxidantes estudiados fueron los taninos, flavonoides y fenoles totales, así como su actividad antioxidante mediante cuatro métodos (ORAC$\mathrm{H}$, FRAP, DPPH y ABTS).

\section{MATERIALES Y MÉTODOS}

Los materiales y métodos se presentan en once subsecciones donde se describe el proceso de tostión realizado en el café, además del desarrollo de los análisis fisicoquímicos y sensoriales. Finalmente, se detalla la realización de los análisis de compuestos antioxidantes y su actividad antioxidante.

\section{Materias primas}

Se evaluaron muestras de café pergamino (Coffea arabica L. var. Castillo), proveniente del municipio de Anserma del departamento de Caldas, de la finca "San Rafael", ubicada a una altura promedio de 1750 m.s.n.m., a una temperatura de $20^{\circ} \mathrm{C}$ y una humedad relativa del $72 \%$ en promedio. Se procesaron $1500 \mathrm{~g}$ de cada muestra por triplicado para todas las determinaciones realizadas.

\section{Análisis fisicoquímicos}

Se separó el pergamino del grano verde mediante un proceso de trillado (Quantik, CR-2000, Armenia, Colombia). Las muestras se tamizaron según la norma técnica colombiana ICONTEC (NTC) 5248 (2013). Para los ensayos sólo se empleó el café retenido sobre la malla de 14 / 64 pulgadas (Equivalente a un café 
excelso U.G.Q.). El café fue despasillado eliminando los defectos del grupo I y II. El contenido de humedad de los granos verdes se determinó en una estufa de convección forzada (Dies, TH115FM, Antioquia, Colombia). El proceso de tostión se realizó a $180^{\circ} \mathrm{C}$ y $100 \%$ de potencia, en un tostador a escala de laboratorio (Quantik, TC-150 A/R, Armenia, Colombia). Se tomaron muestras cada 30 segundos a partir del minuto 2 hasta el minuto 9 por triplicado. La molturación de las muestras tostadas se realizó en un molino (Grindmaster 810, México) a un grado medio.

El tamaño de partícula del material molido se verificó con la serie Taylor, establecido en el procedimiento descrito en la NTC 2441 (2011). El color de las muestras tostadas y molidas se determinó en un colorímetro digital (Quantik, IR800, Armenia, Colombia) expresado como coordenada $L^{*}$ (descriptor de la luminosidad) según la ecuación 1. El pH de las muestras se determinó mediante un pH-metro digital (Lab-850; Schott Instruments ${ }^{\circledR}$, Alemania). Los sólidos solubles se midieron por refractometría y se expresaron como grados Brix ( ${ }^{\circ}$ Brix) con un refractómetro digital (PAL-1, Atago $\AA^{\circledR}$, Japón) (Puerta, 2012). La pérdida de peso (\% merma) se determinó en una balanza de precisión (Fénix-Plus, Bogotá, Colombia) (Caicedo-Eraso et al., 2016). Las muestras se empacaron herméticamente en bolsas de polietileno de alta densidad y se almacenaron a una temperatura de $4{ }^{\circ} \mathrm{C}$ para su posterior preparación y análisis.

$$
\mathrm{L}=0,0608 \text { (Valor colorímetro Quantik) +5,0209 }
$$

En la realización de la prueba de taza de las muestras evaluadas (tomadas en los minutos del 2 al 9 cada 30 segundos por triplicado), se prepararon las respectivas bebidas de acuerdo a la NTC 3566 (2011). Las muestras fueron evaluadas con la prueba de perfil de sabor Q.D.A (Análisis sensorial cuantitativo descriptivo), proporcionando una calificación por catador (3 catadores expertos). Los atributos evaluados en cada muestra fueron: fragancia, aroma, acidez, amargo, cuerpo e impresión global. Adicionalmente se tuvieron en cuenta otros descriptores en la evaluación sensorial de las bebidas para describir la presencia de olores o sabores extraños que representen el deterioro del producto, contaminación o defectos de acre, vinagre, fermento, astringencia, reposo, rancidez, fenólico, terroso, o químico y otros más. Los datos de las pruebas de taza Q.D.A. se convirtieron en gráficas radiales.

\section{Preparación de muestra}

En cada muestra de café tostado y molido se empleó agua destilada como solvente para aislar la mayor cantidad de analito. Los extractos fueron enfriados y diluidos a concentraciones fijas para realizar las medidas de capacidad antioxidante y sustancias químicas antioxidantes (Rojano et al., 2015).

\section{Evaluación de la capacidad de absorción de radicales de oxígeno (ORAC-H)}

En las determinaciones ORAC-H se empleó 6-hidroxi-2,5,7,8-tetrametilcromo-2-ácido carboxílico o Trolox (Merck (Alemania) como estándar y condiciones controladas de temperatura a $37^{\circ} \mathrm{C}$ y pH 7,4 . Las lecturas se realizaron a una longitud de onda (I) de excitación de $493 \mathrm{~nm}$ y una apertura de excitación 5 , I de emisión $515 \mathrm{~nm}$ y apertura de emisión 13, con atenuador de $1 \%$ y sin placa atenuadora. Se utilizaron soluciones de fluoresceína de $1 \times 10^{-2} \mathrm{M}$ en solución buffer de fosfato (PBS) (75 mM), 2,2'-Azinobis (2-amidinopropano) diclorhidrato (AAPH) 0,6 M en PBS (75 mM). Las muestras de café tostado a diferentes tiempos se prepararon con $21 \mu \mathrm{L}$ de fluoresceína, $2,899 \mu \mathrm{L}$ de PBS, $30 \mu \mathrm{L}$ del extracto ensayado y $50 \mu \mathrm{L}$ de AAPH. Se usó Trolox como referencia. El efecto protector del antioxidante se calculó usando las diferencias de áreas bajo la curva de decaimiento de la fluoresceína entre el blanco y la muestra, y se comparó contra la curva del Trolox, y se expresó en $\mu$ mol Trolox / $100 \mathrm{~g}$ muestra o TEAC m, de acuerdo con la ecuación 2 (Zapata et al., 2013). Las pruebas se realizaron por triplicado. La disminución en la intensidad de la fluorescencia fue realizada en un espectrofluorímetro (Perkin-Elmer LS-55, Beaconstield, UK). La fluoresceína, el PBS, el fosfato ácido de sodio y el AAPH se adquirieron en la casa comercial Aldrich Chem. Co (Millwakee, WI).

$$
\text { ORAC } \left.-\mathrm{H}=\frac{\mathrm{AUC}_{\text {muestra }}-\mathrm{AUC}_{\text {control }}}{\mathrm{AUC}_{\text {trolox }}-\mathrm{AUC}_{\text {control }}} . \text { f. [trolox }\right]
$$

Donde, $A \cup C_{\text {muestra }}$ es el área bajo la curva de la muestra, $\mathrm{AUC}_{\text {control }}$ área bajo la curva para el control, $\mathrm{AUC}_{\text {Trolox }}$ área bajo la curva para el Trolox, f es el factor de dilución de los extractos.

\section{Evaluación del poder antioxidante reductor del hierro (FRAP)}

Un volumen de $10 \mu \mathrm{L}$ de las muestras de extracto de café en diferentes concentraciones, se mezcló con 90 $\mu \mathrm{L}$ de agua destilada y $900 \mu \mathrm{L}$ del reactivo FRAP $(2,5 \mathrm{~mL}$ de la solución 2,4,6-tripiridil-s-triazina (TPTZ) a una 
concentración de $10 \mu \mathrm{M}$ en $\mathrm{HCl} 40 \mathrm{mM} ; 2,5 \mathrm{~mL}$ de $\mathrm{FeCl}_{3} 20 \mu \mathrm{M}$ y $25 \mathrm{~mL}$ de buffer acetato $0,3 \mu \mathrm{M}$ a un pH de 3,6). La absorbancia fue leída a $593 \mathrm{~nm}$ después de $7 \mathrm{~min}$. Se empleó una curva de calibración de ácido ascórbico (AA) y las actividades de los extractos de café tostado a diferentes tiempos se expresaron como $\mathrm{mg}$ de AA / $100 \mathrm{~g}$ de muestra (Zapata et al., 2013). Los valores se expresaron como la media de tres réplicas. Como control positivo se empleó el antioxidante AA (Merck, Alemania). El HCl, el TPTZ reductor de $\mathrm{Fe}^{3+}$, el $\mathrm{FeCl}_{3}$ se adquirieron con la casa comercial Aldrich Chem. Co (Millwakee, WI). Para estos ensayos se empleó un espectrofotómetro UV-VIS (Jenway, 6405, Essex, Inglaterra).

\section{Determinación de la capacidad antioxidante DPPH}

También conocida como actividad atrapadora del radical libre DPPH• (1,1-difenil2-picrilhidrazilo), por medio de la disminución en la absorbancia leída, luego de 30 min de reacción a una longitud de onda de $517 \mathrm{~nm}$. El ensayo se llevó a cabo utilizando $100 \mu \mathrm{L}$ de extracto de las muestras tostadas a diferentes tiempos y $900 \mu \mathrm{L}$ de la solución de DPPH . Como referencia del reactivo, se usó la misma cantidad de DPPH • y $100 \mu \mathrm{L}$ del solvente de la muestra. Luego de 30 minutos de reacción a temperatura ambiente y en la oscuridad, se leyó la absorbancia (A) a $517 \mathrm{~nm}$. Para cada muestra en estudio, se calculó el porcentaje de inhibición del radical y los resultados se expresaron como valores TEAC m (Trolox Equivalent Antioxidant Capacity) ( $\mu$ mol Trolox / $100 \mathrm{~g}$ muestra) del compuesto mediante una curva patrón empleando como antioxidante Trolox (Zapata et al., 2013). El porcentaje de inhibición se calculó con la ecuación 3.

$$
\text { Porcentaje de Inhibición }=\left[1-\left(\frac{A_{\text {muestra }}-A_{\text {blanco muestra }}}{A_{\text {referencia }}-A_{\text {blanco referencia }}}\right)\right] \times 100
$$

Con los porcentajes de inhibición y la concentración se calculó la concentración inhibitoria del 50\% (IC50), expresada como $\mu \mathrm{M}$ del compuesto.

\section{Determinación de la actividad antioxidante ABTS}

Las mediciones se realizaron a una longitud de onda de $734 \mathrm{~nm}$. Se utilizaron $100 \mu \mathrm{L}$ de extracto de las muestras de café tostadas a diferentes tiempos y $900 \mu \mathrm{L}$ de la solución del radical ABTS•+. Luego de 60 minutos de reacción a temperatura ambiente y en la oscuridad, se leyó el cambio en la absorbancia respecto a la referencia del reactivo correspondiente a una solución del radical ABTS•+ con el solvente de la muestra. El valor de absorbancia se comparó con la curva de referencia construida con Trolox como patrón primario. Para los dos compuestos se determinó la concentración inhibitoria del $50 \%$ (IC50) mostrada en la ecuación 3, y los resultados se expresaron como valores TEAC m ( $\mu$ mol Trolox / $100 \mathrm{~g}$ muestra). (Zapata et al., 2013).

\section{Taninos condensados}

Se tomaron $230 \mu \mathrm{L}$ de muestra de extracto de café tostado a diferentes tiempos y fueron adicionados a $670 \mu \mathrm{L}$ de una solución de vainillina recién preparada $(1 \mathrm{~g} / 100 \mathrm{ml}$ ) en ácido sulfúrico al $70 \%$. La mezcla se incubó durante $15 \mathrm{~min}$ a $20^{\circ} \mathrm{C}$ y se efectuó la lectura espectrofotométrica a $500 \mathrm{~nm}$ y se comparó con la curva patrón usando como estándar (+)-Catequina. Los resultados se expresaron como mg de Catequina Equivalente / 100g de muestra (Zapata et al., 2013).

\section{Determinación de fenoles totales}

La determinación de fenoles se realizó por el método colorimétrico de Follin-Ciocalteu (Zapata et al., 2013). Se construyó una curva patrón usando como estándar ácido gálico. Los resultados se expresaron como mg de Ácido Gálico / $100 \mathrm{~g}$ de café tostado a diferentes tiempos. Las lecturas se realizaron a 760 $\mathrm{nm}$ por triplicado. Se empleó un espectrofotómetro UV-VIS (Jenway, 6405, Essex, Inglaterra). El reactivo Follin-Ciocalteu y el ácido gálico se obtuvieron de la casa comercial Merck (Alemania).

\section{Contenido de flavonoides totales}

A una alícuota de $0,5 \mathrm{~mL}$ de solución de muestra de café tostado a diferentes tiempos, se le adicionaron $0,5 \mathrm{~mL}$ de solución etanólica de $\mathrm{AlCl}_{3}$ al $2 \%$. Después de una hora de incubación a temperatura ambiente, la absorbancia fue medida a $420 \mathrm{~nm}$. Se emplearon soluciones de catequina (Sigma-Aldrich $\AA_{\text {, USA) entre }}$ 5 - $25 \mu \mathrm{g} / \mathrm{mL}$ con etanol (Merck, Alemania), para la construcción de la curva de calibración. El contenido de flavonoides totales fue calculado como mg equivalentes de catequina / $100 \mathrm{~g}$ muestra. Este procedimiento se efectuó con cada una de las muestras por triplicado. Los valores presentados corresponden a la media y a la desviación estándar ( \pm ). Se utilizó un espectrofotómetro UV-VIS (Jenway, 6405, Essex, Inglaterra). (Zapata et al., 2013) 


\section{Análisis estadísticos}

Todas las determinaciones se realizaron por triplicado y se expresaron los valores como los promedios y la desviación estándar $( \pm)$. Las diferencias estadísticas se determinaron mediante análisis de varianza (ANDEVA) y test de mínima diferencia significativa (LSD), con un valor $\mathrm{P}<0,05$ para comparación de medias de en cada una de las variables analizadas; la actividad antioxidante y el contenido de metabolitos antioxidantes, en las muestras sometidas a tostión a diferentes tiempos. Se utilizó el paquete estadístico SAS. Las regresiones lineales fueron calculadas con un nivel de significancia del 95\% $(P<0,05)$, mediante el paquete estadístico STATGRAPHICS Centurion XV. Se obtuvo una correlación matemática entre la capacidad antioxidante FRAP y el contenido de fenoles totales y flavonoides, con el correspondiente coeficiente de determinación $\left(\mathrm{R}^{2}\right)$.

\section{RESULTADOS Y DISCUSIÓN}

La humedad inicial de las muestras de café verde (trillado y despasillado) fue del $12 \%( \pm 0,24)$. Para garantizar que los resultados obtenidos en ese estudio son comparables entre sí, se emplearon muestras de café de una misma variedad y a un mismo contenido de humedad inicial. De igual manera, al fijar una temperatura de tostión y grado de molienda similar entre las muestras analizadas, se buscó que el nivel de degradación térmica de los compuestos antioxidantes fuera uniforme (Naranjo et al., 2011). Al analizar la cinética de algunas variables fisicoquímicas durante la evolución del proceso de tostión se registraron los siguientes hallazgos (Tabla 1):

a) Se observó que la luminosidad $\left(L^{*}\right)$ de las muestras del café disminuyó con el aumento del tiempo de tostión. La denominación de color de las muestras tostadas entre 4,5 a 6,5 min fue calificada como característica de una tostión clara, mientras que la luminosidad registrada en las muestras tratadas entre 7 y 9 min fue considerada como de tostión oscura. Las coloraciones oscuras se deben a la generación de melanoidinas que aportan tonalidades pardas a negras, producto de las reacciones de caramelización (Lee et al., 2017) Maillard y polimerización (Moreira et al., 2017).

b) El $\mathrm{pH}$ de las muestras descendió con el incremento del tiempo de tostión dado que el agua libre fue evaporada durante el tratamiento térmico, y los ácidos orgánicos presentes en el café se concentraron causando el descenso del pH. Durante el proceso de tostión se generan y se concentran, varios compuestos responsables del aroma y del sabor del café, tales como la cafeína, la trigonelina, los ácidos clorogénicos, el ácido cítrico, el ácido acético y el ácido fórmico presentes en los granos (De Luca et al., 2016; Nguyen y Byun, 2013); los cuales tienen una incidencia directa en este parámetro fisicoquímico.

c) Los sólidos solubles expresados como ${ }^{\circ}$ Brix presentaron los valores más altos entre 2 y 4,5 min de tostión, lo que indicó que el tiempo de tostado incidió sobre la transformación y solubilización de los azúcares, los ácidos orgánicos, las sales y demás compuestos solubles en agua presentes en el café; dado que la modificación estructural del mismo, permite aumentar la solubilidad de sus componentes en agua caliente durante la preparación de las bebidas (Puerta, 2012).

d) El porcentaje de merma de las muestras, aumentó como era de esperarse debido al intenso proceso de evaporación del agua llevado a cabo durante la tostión; donde el agua evaporada aumentó la porosidad del grado de café con un progresivo descenso en el peso de las muestras a mayor tiempo de tostión. El porcentaje de merma industrial está fijado entre un 18 y un $20 \%$ que correspondió a los tiempos de tostión entre 6,5 a 8 min de este estudio. De acuerdo a estudios realizados por De Luca et al. (2016), que coinciden con los obtenidos en este estudio, el grado de tostión del café es un parámetro muy importante para evaluar la calidad del producto final, por esta razón se ha clasificado en tres categorías relacionadas con la pérdida de peso (merma) de los granos: tostión ligera (11\% de pérdida de peso), tostión media (14\%) y tostión oscura (20\%). El análisis de varianza indicó una diferencia significativa $(p<0,05)$ entre las variables evaluadas en la tabla 1 con respecto al tiempo de tostión aplicado.

Las calificaciones dadas a las muestras extraídas a diferentes tiempos de tostión entre 2 y 4,5 min con una frecuencia de $30 \mathrm{~s}$, no coinciden, alejándose de manera importante de la calificación de un café U.G.Q., dado que estos tiempos de tostión aún no han generado ni el color ni los perfiles de taza característicos de un café excelso (U.G.Q.) (resultados no mostrados). De forma general, la duración del proceso de tostión del café es aproximadamente de 5 a 12 min, en función del tipo de tostador empleado y los parámetros del proceso como la temperatura y la potencia (Lee et al., 2017). El segundo grupo de muestras catadas (Figura 1), que comprende tiempos de tostado entre 5 y 7,5 min, tomadas con la misma frecuencia, obtuvo calificaciones cercanas al patrón U.G.Q. La muestra extraída a los 5 min de tostión, no tuvo una calificación igual al patrón y presentó marcadas notas astringentes. No obstante, Las muestras tostadas en 5,5-6 y 6,5 min, presentaron un perfil de taza idéntico al patrón con una óptima calificación en los 7 atributos evaluados. Además, las muestras extraídas entre 7 y 7,5 min presentaron diferencias en la acidez y el amargo y los atributos acidez, amargo y cuerpo, en comparación con la muestra U.G.Q., respectivamente. 
Tabla 1: Parámetros fisicoquímicos de las muestras de café tostadas a diferentes tiempos. Los valores promedio $(n=3)$ con letras diferentes $(a-i)$ en la misma columna indican diferencias estadísticas al nivel de significancia del $5 \%(p<0,05)$.

\begin{tabular}{ccccc}
\hline $\begin{array}{c}\text { Tiempo de } \\
\text { tostión }(\mathrm{min})\end{array}$ & $\begin{array}{c}\text { Color } \mathrm{L}^{*} \\
(\text { Luminosidad })\end{array}$ & $\mathrm{pH}$ & ${ }^{\circ}$ Brix & $\%$ Merma \\
\hline 2,0 & $25,421( \pm 0,39) \mathrm{a}$ & $6,199( \pm 0,17) \mathrm{a}$ & $1,147( \pm 0,55) \mathrm{a}$ & $4,319( \pm 0,48) \mathrm{a}$ \\
2,5 & $23,555( \pm 0,64) \mathrm{a}$ & $6,188( \pm 0,32) \mathrm{a}$ & $1,283( \pm 0,57) \mathrm{a}$ & $4,951( \pm 0,38) \mathrm{a}$ \\
3,0 & $22,582( \pm 0,43) \mathrm{b}$ & $6,182( \pm 0,57) \mathrm{a}$ & $1,374( \pm 0,18) \mathrm{b}$ & $5,389( \pm 0,42) \mathrm{b}$ \\
3,5 & $21,731( \pm 0,22) \mathrm{b}$ & $6,089( \pm 0,27) \mathrm{a}$ & $1,422( \pm 0,23) \mathrm{b}$ & $5,565( \pm 0,16) \mathrm{b}$ \\
4,0 & $21,366( \pm 0,17) \mathrm{b}$ & $6,062( \pm 0,46) \mathrm{a}$ & $2,074( \pm 0,35) \mathrm{c}$ & $9,232( \pm 0,52) \mathrm{c}$ \\
4,5 & $15,529( \pm 0,35) \mathrm{c}$ & $5,993( \pm 0,13) \mathrm{b}$ & $2,225( \pm 0,41) \mathrm{d}$ & $11,539( \pm 0,23) \mathrm{d}$ \\
5,0 & $15,408( \pm 0,56) \mathrm{c}$ & $5,495( \pm 0,68) \mathrm{b}$ & $2,357( \pm 0,45) \mathrm{d}$ & $13,231( \pm 0,17) \mathrm{e}$ \\
5,5 & $14,678( \pm 0,39) \mathrm{c}$ & $5,454( \pm 0,27) \mathrm{b}$ & $2,364( \pm 0,18) \mathrm{d}$ & $14,211( \pm 0,33) \mathrm{e}$ \\
6,0 & $13,658( \pm 0,45) \mathrm{d}$ & $5,374( \pm 0,11) \mathrm{b}$ & $2,533( \pm 0,19) \mathrm{e}$ & $15,337( \pm 0,48) \mathrm{e}$ \\
6,5 & $11,590( \pm 0,64) \mathrm{e}$ & $5,343( \pm 0,49) \mathrm{c}$ & $2,621( \pm 0,60) \mathrm{e}$ & $17,614( \pm 0,55) \mathrm{f}$ \\
7,0 & $9,024( \pm 0,34) \mathrm{f}$ & $5,299( \pm 0,27) \mathrm{c}$ & $2,432( \pm 0,51) \mathrm{d}$ & $17,379( \pm 0,26) \mathrm{f}$ \\
7,5 & $6,166( \pm 0,40) \mathrm{f}$ & $5,235( \pm 0,18) \mathrm{c}$ & $2,464( \pm 0,35) \mathrm{d}$ & $18,688( \pm 0,36) \mathrm{g}$ \\
8,0 & $3,944( \pm 0,53) \mathrm{g}$ & $5,178( \pm 0,56) \mathrm{c}$ & $2,001( \pm 0,25) \mathrm{c}$ & $19,053( \pm 0,12) \mathrm{g}$ \\
8,5 & $2,966( \pm 0,36) \mathrm{h}$ & $5,163( \pm 0,25) \mathrm{c}$ & $1,921( \pm 0,28) \mathrm{c}$ & $21,812( \pm 0,26) \mathrm{h}$ \\
9,0 & $1,991( \pm 0,47) \mathrm{i}$ & $5,077( \pm 0,22) \mathrm{d}$ & $1,725( \pm 0,32) \mathrm{c}$ & $25,037( \pm 0,58) \mathrm{i}$ \\
\hline
\end{tabular}
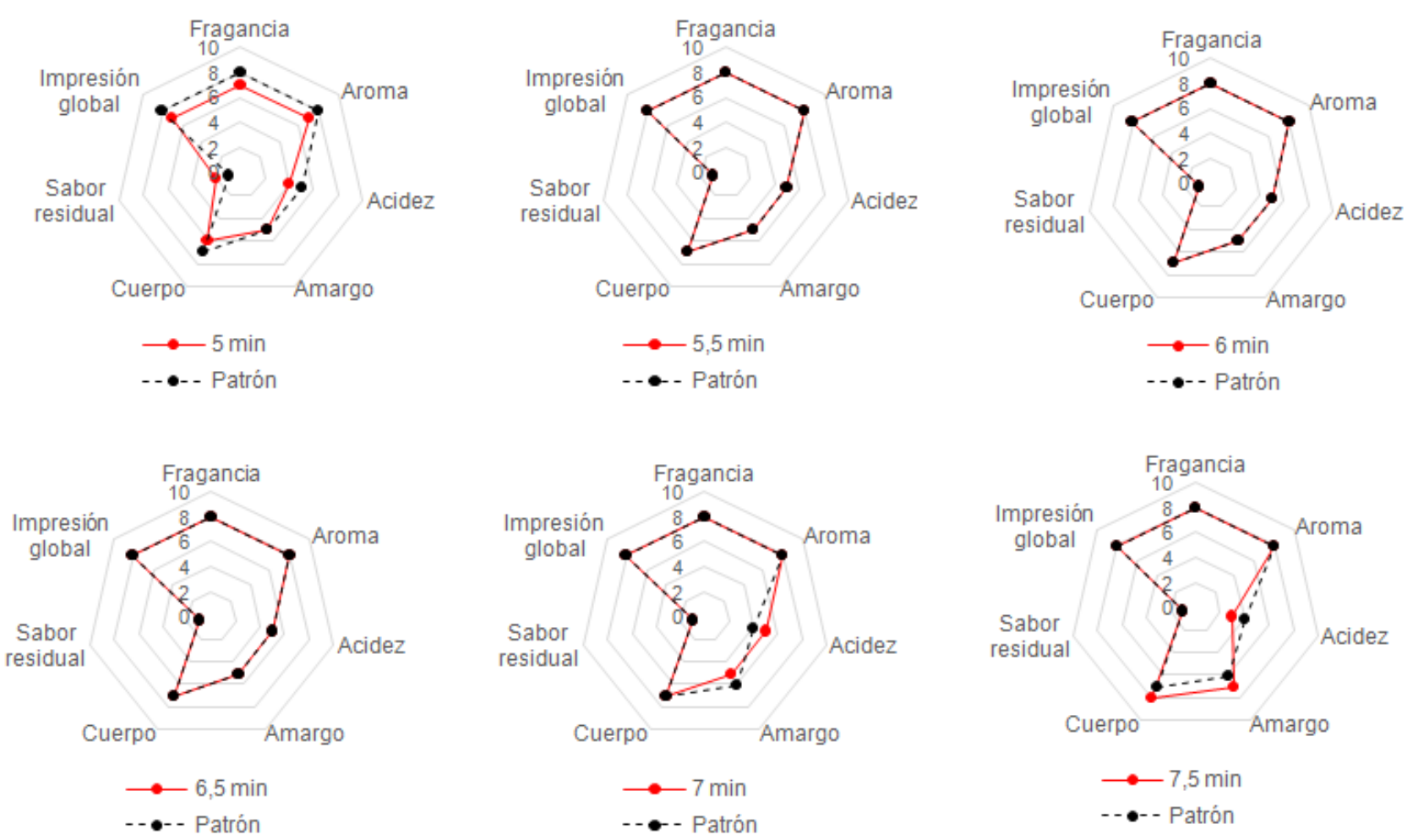

Fig.1: Perfil sensorial de las muestras de café tostado en el minuto $5-5,5-6-6,5-7$ y 7,5 comparado con un patrón U.G.Q. Los valores promedio $(n=3)$.

El tercer y último grupo de muestras catadas (resultados no mostrados) representan los tiempos de tostión final de 8-8,5 y 9 min. La muestra de 8 min presentó diferencias con respecto al patrón, en su acidez, amargo y cuerpo; presentando un comportamiento similar a la muestra extraída en 7,5 min. Con 8,5 min de tostión, la muestra presentó diferencias en los atributos de impresión global, acidez, amargo y cuerpo con respecto al patrón U.G.Q., además, presentó notas amargas y a sobrequemado. En la muestra con 9 min de tostión, se encontró que la mayoría de atributos difiere del patrón y se percibieron marcadas notas a quemado, así como la exudación de gotas de grasa en la superficie del grano. 
El contenido de fenoles totales se incrementó entre 5 y 6,5 min de tostión con valores entre 3300 y $3400 \mathrm{mg}$ de ácido gálico / $100 \mathrm{~g}$ de muestra (Figura 2). Tostiones entre 7 y $9 \mathrm{~min}$, registraron que el contenido de fenoles totales decreció considerablemente, mostrando un efecto significativo del tiempo de tostión sobre la retención de fenoles totales $(p<0,05)$. Esto indica que la tostión a grado medio es favorable para obtener café tostado con una alta concentración de fenoles totales. De acuerdo a los hallazgos de Naranjo et al. (2011), el café calidad Excelso (U.G.Q.) contiene altas concentraciones de ácidos fenólicos responsables de su mayor actividad antioxidante, comparado con otras calidades de café colombianos. En concordancia con los resultados obtenidos, Kwak et al. (2017) determinaron que las altas temperaturas disminuyeron la retención de los fenoles totales en café tostado $\left(180-200^{\circ} \mathrm{C}\right)$ aplicando aire caliente. De igual manera, Somporn et al. (2011) indicaron que el contenido de polifenoles decrece al aumentar la intensidad del tostado del café (ligera a oscura). Además, la descomposición térmica de los fenoles durante la tostión da paso a la liberación de la cafeína en el grano, y ésta última, contribuye al perfil de taza de la bebida de café, al conferir cuerpo, fuerza y notas amargas a la bebida (Cheng et al., 2016); no obstante, el contenido de cafeína puede disminuir al aumentar la intensidad del tratamiento térmico o la duración de la tostión (Hečimović et al., 2011).

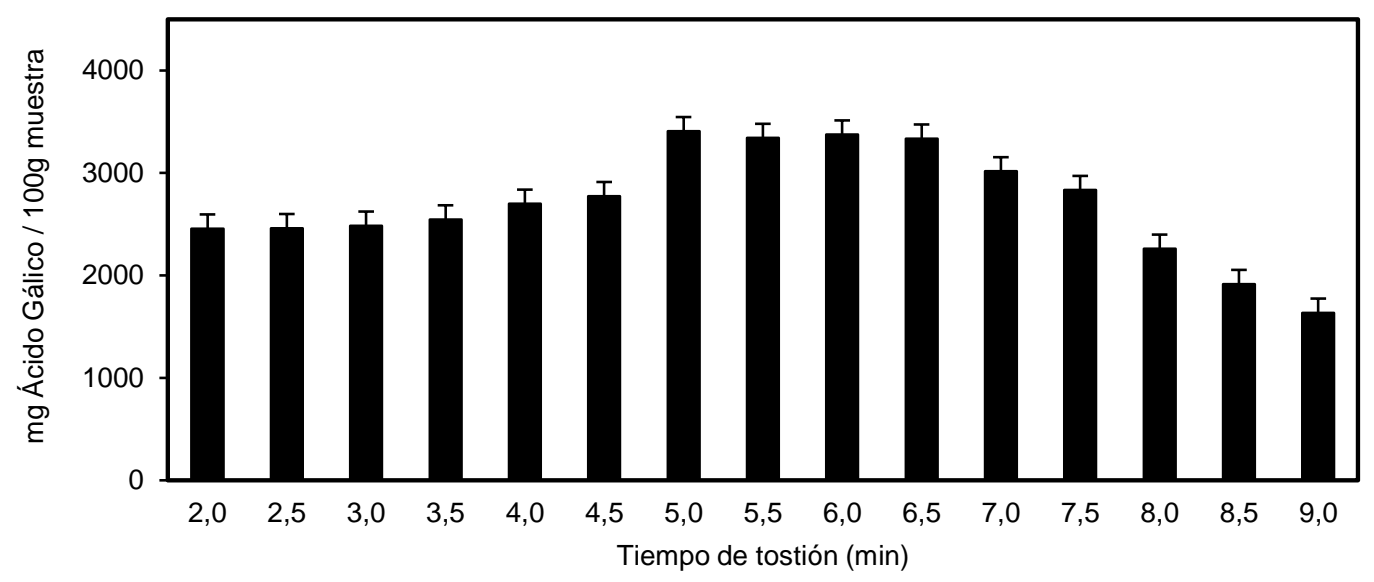

Fig. 2: Contenido de fenoles totales durante el proceso de tostión a diferentes tiempos.

El aumento en el contenido de fenoles durante los tiempos intermedios de tostión, está relacionado directamente con el procesamiento térmico que interviene en la síntesis de productos secundarios de la reacción de Maillard (Moreira et al., 2017). Algunos de estos compuestos secundarios son las melanoidinas o compuestos de color marrón que poseen actividad antioxidante, generan sabor, color y tienen propiedades reductoras (Lee et al., 2017). De acuerdo a otros estudios, las mayores variaciones cuantitativas de compuestos químicos durante la tostión han sido reportadas en los compuestos fenólicos dado que las condiciones de $\mathrm{pH}$ hace que generen productos de las reacciones de transesterificación como la cafeína, así como un aporte importante de otros compuestos fenólicos como el ácido clorogénico y el cafeico con propiedades antioxidantes (Hečimović et al., 2011). Finalmente, Ross et al. (2011) determinaron que los compuestos fenólicos tienen un carácter termosensible comparado con su actividad antioxidante (tres veces menor), formados en el proceso de tostado por el rompimiento y transformación de los ácidos clorogénicos. Estos últimos, son determinantes en el perfil de taza, porque durante la tostión forman una variada gama de compuestos afectados por el grado de tostión y procesamiento del café. Los ácidos clorogénicos aportan acidez, astringencia y notas amargas a la bebida (Cheng et al., 2016). Su degradación durante la tostión (60$98 \%$ ), guarda estrecha relación con el aumento de la concentración de ácido quínico, así como con la degradación térmica del ácido hidroxicinámico y algunos compuestos fenólicos durante el proceso de tostión (Lee et al., 2015). El consumo frecuente de fenoles en la dieta en dosis definidas, regula el estrés oxidativo y ayuda a proteger al organismo de las enfermedades cardiovasculares, así como de diabetes mellitus y de algunas enfermedades crónicas, de igual forma, se han comprobado sus propiedades antimicrobianas, antiinflamatorias y antialergénicas (Mussatto, 2015).

La concentración de flavonoides aumentó entre 5 y 6,5 min de tostión, con un máximo de concentración del orden de $3290 \mathrm{mg}$, expresados como $\mathrm{mg}$ de catequina equivalente / $100 \mathrm{~g}$ muestra (Figura 3). El contenido de flavonoides fue bajo de 8 a 9 min debido a la prolongación de los tiempos de tostión, indicando un efecto significado $(p<0,05)$ del tiempo de procesamiento térmico sobre la retención de flavonoides en café tostado. Las tostiones medias permiten una alta retención de flavonoides en el grano de café tostado y los perfiles sensoriales de 5,5 a 6,5 min indicaron un perfil de taza igual al del café U.G.Q. Además de la cafeína y los ácidos clorogénicos, los flavonoides son responsables de conferir sabor amargo al café por su contenido de fenoles en su estructura. No obstante, es importante aclarar que también el proceso de tostión puede conferir sabor amargo al café, así pues para tostiones oscuras aumenta el sabor amargo de la bebida, mientras que para tostiones claras se generan notas ácidas (Cheng et al., 2016). Los efectos de este compuesto 
antioxidante sobre la salud, incluyen reportes como anticancerígeno, antinflamatorio y antiarterosclerótico; asimismo, ayuda a prevenir enfermedades como el Alzheimer y el Parkinson (Chen y Chen, 2013; KurzawaZegota et al., 2012). Los flavonoides también contribuyen a la reducción de la prevalencia de enfermedades coronarias y ayudan al equilibrio microbiano a nivel intestinal (Cardona, et al, 2013).

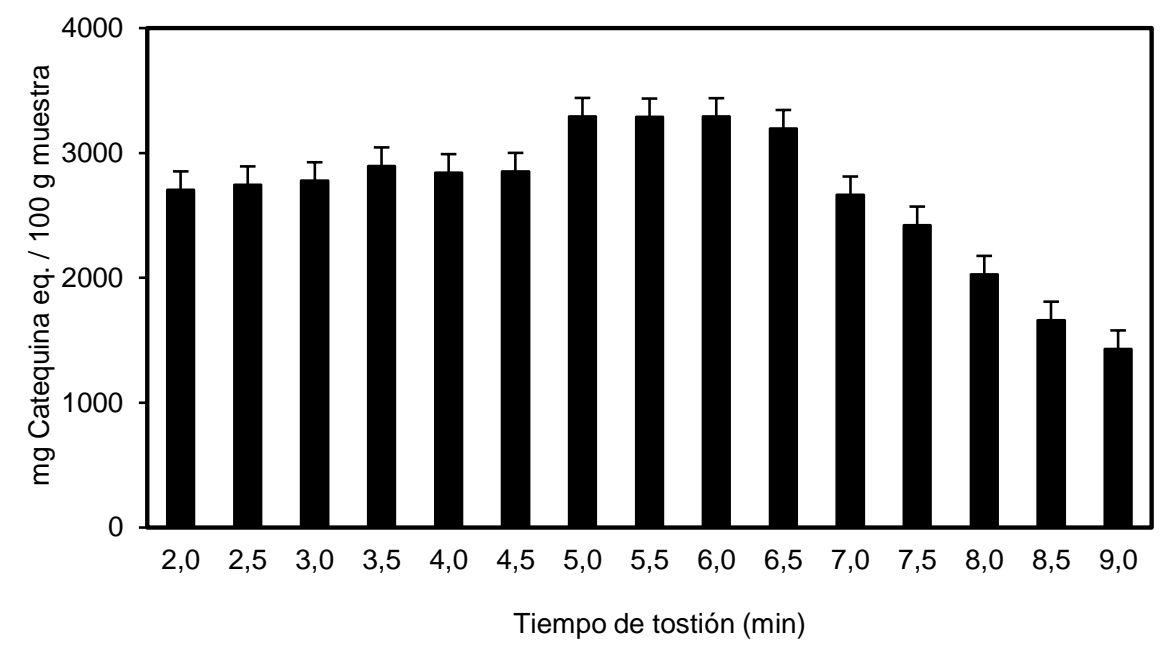

Fig. 3: Contenido de flavonoides durante el proceso a de tostión a diferentes tiempos.

Con relación a la retención de taninos en las muestras de café tostadas a diferentes tiempos (Figura 4), se encontró que este compuesto no se degradó térmicamente a tiempos mayores de tostión, por el contrario, se incrementó su concentración conforme aumentó la duración del tostado con una concentración máxima de $495 \mathrm{mg}$ expresados como mg de catequina eq. / 100g de muestra. El análisis estadístico indicó una diferencia significativa $(p<0,05)$ en la concentración de taninos condensados con respecto al tiempo de tostión. El incremento en la concentración de taninos en café se acentúa con las tostiones oscuras y en la prueba de taza está estrechamente relacionado con notas de astringencia. Con respecto a los resultados obtenidos, la concentración de taninos alcanzada entre el min 5,5 a 6,5, presentaron notas de astringencia en un intervalo óptimo, dado que su presencia en la muestra no alteró la calificación sensorial al presentar una calificación igual al del patrón U.G.Q. (Figura 1).Desde el punto de vista biofuncional, los taninos tienen propiedades antiinflamatorias y antihemorrágica y su acción antioxidante ayuda a prevenir el envejecimiento prematuro, así como enfermedades degenerativas (Zapata et al., 2013).

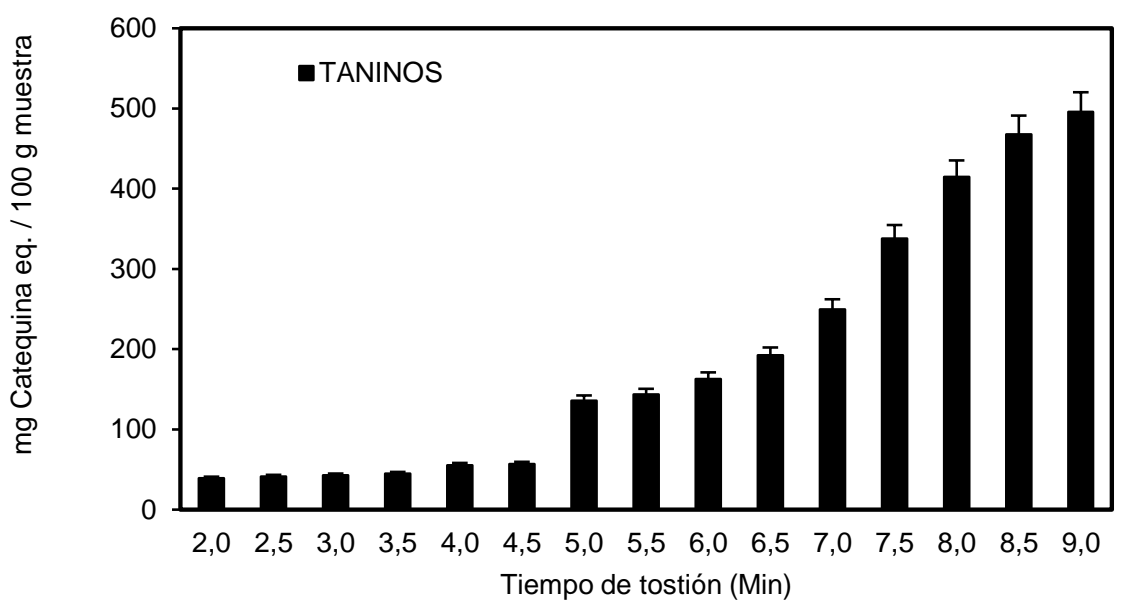

Fig. 4: Contenido de taninos durante el proceso a de tostión a diferentes tiempos.

Los valores obtenidos de actividad antioxidante con los métodos método DPPH, ABTS y ORAC-H y el método FRAP fueron estadísticamente diferentes $(p<0,05)$ (Figura 5 y 6 , respectivamente). Los valores más altos de actividad antioxidante correspondieron con los tiempos de tostión entre el minuto 5 y 6,5 para las 4 metodologías evaluadas, donde las muestras tostadas entre 5,5 y 6,5 min, coincidieron con el perfil de taza del patrón U.G.Q. Estos resultados indicaron una correlación positiva entre la mayor actividad antioxidante y el mejor perfil sensorial alcanzado, únicamente con tostiones suaves (5,5 a 6,5 $\mathrm{min}$ ). A tiempos mayores de tostión se observó un descenso en la expresión de los metabolitos antioxidantes en los 4 métodos estudiados. 


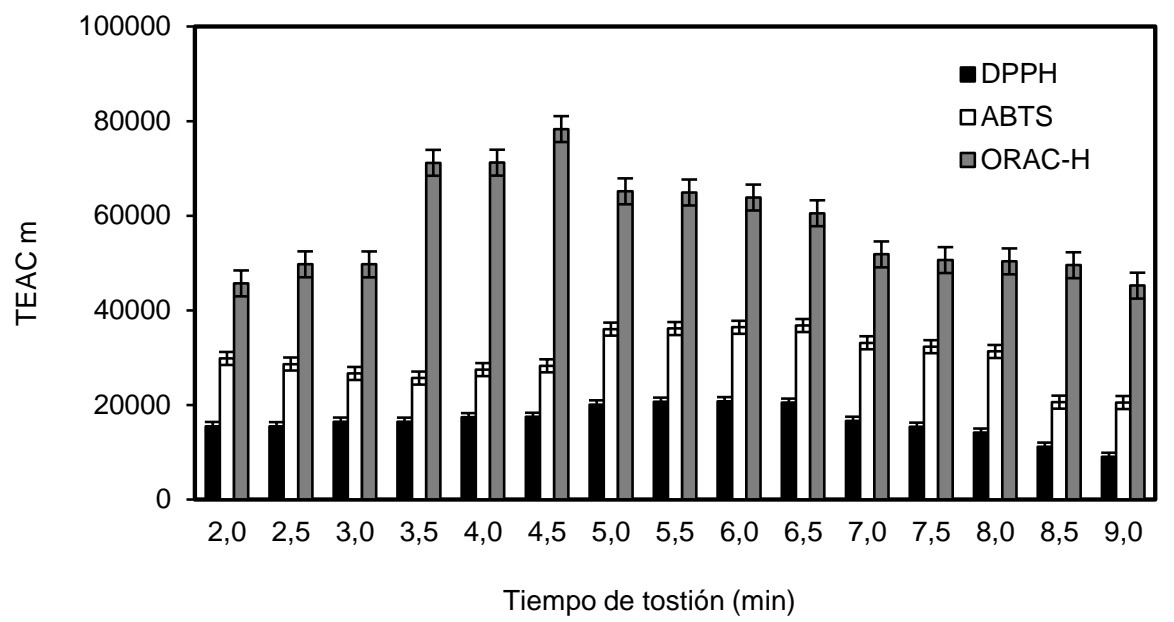

Fig. 5: Capacidad antioxidante por los métodos DPPH, ABTS y ORAC-H durante el proceso a de tostión a diferentes tiempos.

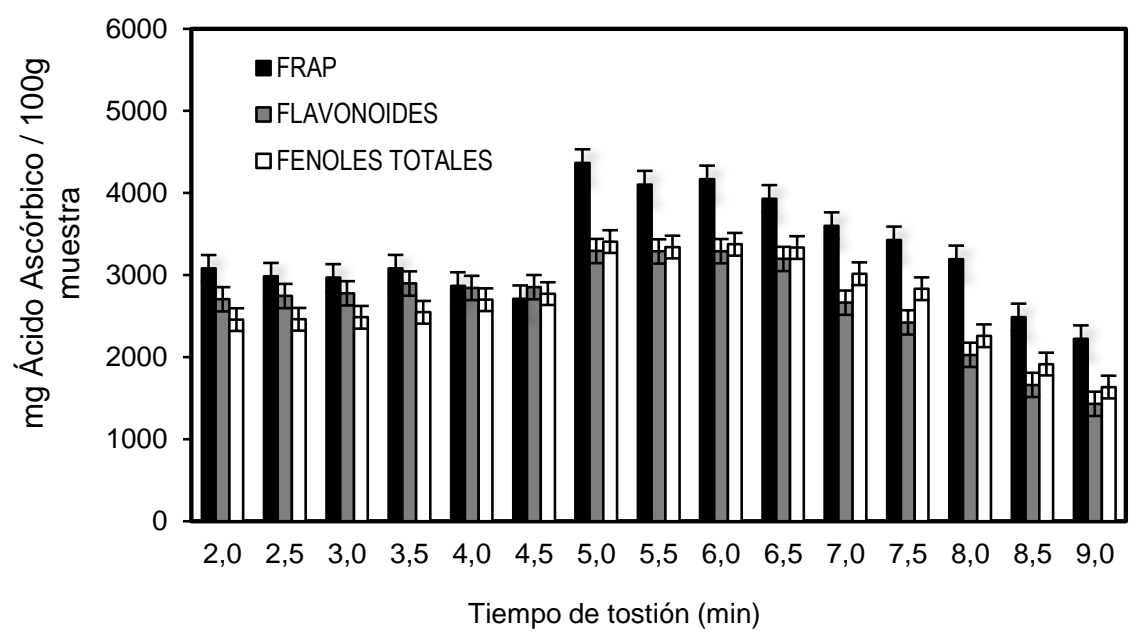

Fig. 6: Capacidad antioxidante FRAP correlacionada con la concentración entre flavonoides y fenoles totales durante el proceso a de tostión a diferentes tiempos.

La correlación entre flavonoides, fenoles totales y la actividad antioxidante por el método FRAP es mostrada en la figura 6, en la cual se observó claramente el mismo comportamiento en las tres variables por efecto del tiempo de tostión aplicado en un café grado U.G.Q. Fonseca-García et al. (2014), estudiaron la capacidad antioxidante de cafés comerciales de Norte de Santander dentro del rango de $3095( \pm 0,000)$ a $6857( \pm 8,115)$ $\mu \mathrm{mol}$ Trolox/g café por el método FRAP, valores que son similares a los reportados en este estudio (Figura 6). Por último, se obtuvo una ecuación generalizada del comportamiento de la actividad antioxidante FRAP correlacionada con el contenido de flavonoides y fenoles totales (Figura 6); representada en la ecuación 4 mediante un polinomio de sexto grado, con un coeficiente de determinación $\mathrm{R}^{2}=0,9907$, donde se infiere que ambos metabolitos aportan de manera significativa a la capacidad antioxidantes de las muestras estudiadas.

$$
y=-0,0129 x^{6}+0,7118 x^{5}-14,656 x^{4}+138,4 x^{3}-608,35 x^{2}+1185,6 x+1979,7
$$

En correspondencia con los resultados de este estudio, Kwak et al. (2017), determinaron el efecto negativo del incremento de la temperatura del tostado de café $\left(180-200^{\circ} \mathrm{C}\right)$ sobre la actividad antioxidante DPPH y ORAC haciendo pasar un flujo de aire durante el proceso de torrefacción y propusieron aplicar tostiones en café combinando temperaturas bajas y largos tiempos o procesos que involucren temperaturas altas y cortos tiempos, para garantizar una alta actividad antioxidante. De igual forma, Somporn et al. (2011) indicaron que la actividad DPPH decreció en café tostado, pasando de tostados ligeros a oscuros, es decir con el incremento del tiempo y de la temperatura de torrefacción simultáneamente. De acuerdo con Vignoli et al. (2011), los compuestos químicos que potencian la actividad antioxidante en el café se generan en parte durante el proceso de tostión, a partir de la reacción de Maillard, así como por compuestos fenólicos pertenecientes al grupo de los ácidos hidroxicinámicos (clorogénico, cafeico, cumárico y ferúlico), melanoidinas, cafeína y algunos de los componentes volátiles del café. De este modo, se confirma la existencia de una correlación directa entre el pardeamiento no enzimático y la capacidad antioxidante del café tostado colombiano (Contreras-Calderón et al. 2016). 


\section{CONCLUSIONES}

El proceso de tostión denominado como grado medio en muestras de café excelso (U.G.Q.), aumentó el contenido de metabolitos secundarios (fenoles totales, flavonoides y taninos condensados) y la actividad antioxidante (ORAC-H, ABTS, DPPH y FRAP), combinado con una calificación de perfil de taza óptima para tiempos de tostión entre 5,5 y $6,5 \mathrm{~min}\left(180^{\circ} \mathrm{C}, 100 \%\right.$ de potencia), otorgándole al café obtenido un equilibrio entre calidad sensorial y propiedades nutracéuticas.

\section{AGRADECIMIENTOS}

Al laboratorio de Ciencia de los Alimentos de la Universidad Nacional de Colombia sede Medellín y al laboratorio de Análisis sensorial e industrialización del café de la Universidad de Caldas por el apoyo técnicocientífico en la preparación y ejecución del presente estudio.

\section{REFERENCIAS}

Aguiar, J., B.N. Estevinho y L. Santos, Microencapsulation of natural antioxidants for food application e the specific case of coffee antioxidants, Trends Food Sci. Tech., ISSN: 0924-2244, 58, 21-39 (2016)

Arango, M., El beneficio ecológico del café en Colombia, Pontificia Universidad Javeriana, Colombia (2012)

Ballesteros, L.F., M.J. Ramirez y otros tres autores, Optimization of autohydrolysis conditions to extract antioxidant phenolic compounds from spent coffee grounds, J. Food Eng., ISSN: 0260-8774, 199, 1-8 (2017)

Caicedo-Eraso, J.C., F.O.A. Díaz y D.R. Varón-Serna, Espectroscopia de impedancia eléctrica aplicada durante la fermentación húmeda del café, Vitae, ISSN: 0121-4004, 23(1), S195-S198 (2016)

Cano-Marquina, A., J.J. Tarín y A. Cano, The impact of coffee on health, Maturitas, ISSN: 0378-5122, 75(1), 7-21 (2013)

Cardona, F., C. Andrés-Lacueva y otros tres autores, Benefits of polyphenols on gut microbiota and implications in human health, The Journal of Nutritional Biochemistry, ISSN: 0955-2863, 24(8), 1415-1422 (2013)

Chen, A.Y. e Y.C. Chen, A review of the dietary flavonoid, kaempferol on human health and cancer chemoprevention, Food Chem., ISSN: 0308-8146, 138(4), 2099-2107 (2013)

Cheng, B., A. Furtado y otros 2 autores, Influence of genotype and enviroment on coffee quality, doi: org/10.1016/j.tifs.2016.09.003, Trends Food Sci. Tech., 57, 20-30 (2016)

Coelho, C., M. Ribeiro y otros cinco autores, Nature of phenolic compounds in coffee melanoidins, J. Agric. Food Chem., ISSN: 0021-8561, 62, 7843-7853 (2014)

Contreras-Calderón, J., D. Mejía-Díaz y otros seis autores, Evaluation of antioxidant capacity in coffees marketed in Colombia: Relationship with the extent of non-enzymatic browning, Food Chem., ISSN: 0308-8146, 209, 162-170 (2016)

De Luca, S., M. De Filippis y otros cuatro autores, Characterization of the effects of different roasting conditions on coffee samples of different geographical origins by HPLC-DAD, NIR and chemometrics, Microchem. J., ISSN: 0026265X, 129, 348-361 (2016)

Farah, A., Coffee constituents. Coffee: Emerging health effects and disease prevention, Wiley-Blackwell, pp. 21-58. Illinois, USA (2012)

FNCC, Comportamiento de la industria Cafetera Colombiana, Federación Nacional de Cafeteros de Colombia (2014)

Fonseca-García, L., L.S. Calderón-Jaimes y M.E. Rivera, Capacidad antioxidante y contenido de fenoles totales en café y subproductos del café producido y comercializado en Norte de Santander (Colombia), Vitae, ISSN: 0121-4004, 21(3) 228-236 (2014)

Gamboa P.Y.R., S. A. S. Mosquera e I. E. N. Paz, Caracterización de taza de café especial en el municipio de chachagüí, departamento de Nariño, Colombia, Biotecnología en el Sector Agropecuario y Agroindustrial, ISSN: 1692-3561, 11(2), 85-92 (2013)

Hečimović, I., A. Belščak-Cvitanović y otros dos autores, Comparative study of polyphenols and caffeine in different coffee varieties affected by the degree of roasting, Food Chem., ISSN: 0308-8146, 129, 991-1000 (2011)

Illy -El café filtro, o café americano: cómo se prepara, Illy, Italia (2017)

Kwak, H.S., S. Ji e Y. Jeong, The effect of air flow in coffee roasting for antioxidant activity and total polyphenol content, Food Control, ISSN: 0956-7135, 71, 210-216 (2017)

Kurzawa-Zegota, M., M. Najafzadeh y otros dos autores, The protective effect of the flavonoids on food-mutagen-induced DNA damage in peripheral blood lymphocytes from colon cancer patients, Food Chem. Toxicol., ISSN: 0278-6915, 50(2), 124-129 (2012)

Lee, L.W., G.Y. Tay y otros cuatro autores, Modulation of the volatile and non-volatile profiles of coffee fermented with Yarrowialipolytica: II. Roasted coffee, doi: org/10.1016/j.Iwt.2017.01.070, LWT--Food Sci. Technol., 80, 32-42 (2017) 
Lee, L.W., M.W. Cheong y otros tres autores, Coffee fermentation and flavor e an intricate and delicate relationship, Food Chem., ISSN: 0308-8146, 185, 182-191 (2015)

Moreira, A.S.P., F.M. Nunes y otros cinco autores, Transglycosylation reactions, a main mechanism of phenolics incorporation in coffee melanoidins: Inhibition by Maillard reaction, Food Chem., ISSN: 0308-8146, 227, 422-431 (2017)

Mussatto, S.I., Generating biomedical polyphenolic compounds from spent coffee or silverskin, Coffee in Health and Disease Prevention, Elsevier, pp. 93-106, London (2015)

Nguyen, T.N.H. y S.Y. Byun, Combined changes of process conditions improved aromatic properties of Vietnamese Robusta, Biotechnol. Bioprocess Eng., ISSN: 1226-8372, 18, 248-256 (2013)

NTC 2441: 2011, ICONTEC, Café tostado y molido. Método para la determinación del tamaño de partícula por distribución granulométrica, pp. 1-9, Bogotá D.C., Colombia (2011)

NTC 5248: 2013. ICONTEC, Café verde. Análisis del tamaño. Tamizado manual y mecánico, pp. 1-15, Bogotá D.C., Colombia (2013)

Oliveros, N.O., J.A. Hernández y otros tres autores, Experimental study of dynamic porosity and its effects on simulation of the coffee beans roasting, J. Food Eng., ISSN: 0260-8774, 199, 100-112 (2017)

Oroian, M. y I. Escriche, Antioxidants: Characterization, natural sources, extraction and analysis, Food Res. Int., ISSN: 0963-9969, 74, 10-36 (2015)

Puerta, G. Factores, controles y procesos en la fermentación del café, Avances Tecnológicos Cenicafé, ISSN: 0120-0178, 1-12 (2012)

Rojano, B., I. Zapata y J. Sepúlveda, Efecto del tiempo de almacenamiento sobre las propiedades fisicoquímicas, probióticas y antioxidantes de yogurt saborizado con mortiño (Vaccinium meridionale Sw), doi:org/10.4067/S071807642015000200004, Información Tecnológica, 26(2), 17-28 (2015)

Ross, C.F., C. Jr. Hoye y V.C. Fernández-Plotka, Influence of heating on the polyphenolic content and antioxidant activity of grape seed flour, J. Food Sc., ISSN: 1750-3841, 76, C884-C890 (2011)

Somporn, C., A. Kamtuo y otros dos autores, Effects of roasting degree on radical scavenging activity, phenolics and volatile compounds of Arabica coffee beans (Coffea arabica I. cv. Catimor), Int. J. Food Sci. Tech., ISSN:1365-2621, 46, 2287-2296 (2011)

Vignoli, J.A., D.G. Bassoli y M.T. Benassi, Antioxidant activity, polyphenols, caffeine and melanoidins in soluble coffee: The influence of processing conditions and raw material, Food Chem., ISSN: 0308-8146, 124, 863-868 (2011)

Yashin, A., Y. Yashin y otros dos autores, Antioxidant and antiradical activity of coffee, Antioxidants, ISSN: 2076-3921, 2(4), 230-245 (2013)

Zapata, K., F.B. Cortés y B.A. Rojano, Polifenoles y Actividad Antioxidante del Fruto de Guayaba Agria (Psidium araca), doi: 10.4067/S0718-07642013000500012, Información Tecnológica, 24(5), 103-112 (2013) 
